\title{
Stability Analysis for Nonlinear Impulsive Control System with Uncertainty Factors
}

\author{
Zemin Ren $\mathbb{D}^{1},{ }^{1}$ Shiping Wen, ${ }^{2}$ Qingyu Li, ${ }^{1}$ Yuming Feng $\mathbb{D},{ }^{3}$ and Ning Tang ${ }^{3}$ \\ ${ }^{1}$ School of Mathematics, Physics and Data Science, Chongqing University of Science and Technology, Chongqing 401331, China \\ ${ }^{2}$ Australian AI Institute, University of Technology Sydney, Ultimo, NSW 2007, Australia \\ ${ }^{3}$ Key Laboratory of Intelligent Information Processing and Control, Chongqing Three Gorges University, Wanzhou, \\ Chongqing 404100, China \\ Correspondence should be addressed to Yuming Feng; yumingfeng25928@163.com
}

Received 13 August 2020; Revised 9 September 2020; Accepted 6 November 2020; Published 21 November 2020

Academic Editor: Michele Migliore

Copyright ( $) 2020$ Zemin Ren et al. This is an open access article distributed under the Creative Commons Attribution License, which permits unrestricted use, distribution, and reproduction in any medium, provided the original work is properly cited.

Considering the limitation of machine and technology, we study the stability for nonlinear impulsive control system with some uncertainty factors, such as the bounded gain error and the parameter uncertainty. A new sufficient condition for this system is established based on the generalized Cauchy-Schwarz inequality in this paper. Compared with some existing results, the proposed method is more practically applicable. The effectiveness of the proposed method is shown by a numerical example.

\section{Introduction}

Impulse control is based on impulsive differential equation and has many applications [1-6], such as digital communication system, artificial intelligence, and financial sector. In comparison with other methods, impulse control is more efficient in dealing with the stability of complex systems. The stability is an important property of the impulsive control system. Mathematically, its goal is to stabilize an unstable system by proper impulse. Up to now, a wide variety of achievements of impulse control theory have been developed in the literature [7-13].

Generally, there are at least one "impulsively" changeable state variable appearing in a plant $P$, which could be described as following control system:

$$
\left\{\begin{array}{l}
\dot{x}(t)=A x+\phi(x), t \neq \tau_{k}, \\
\Delta x=U(k, x), t=\tau_{k}, \quad k=1,2, \ldots, \\
x\left(t_{0}\right)=x_{0} .
\end{array}\right.
$$

Here, $x \in \mathbb{R}^{n}$ denotes the state variable and $U(k, x)$ the impulse control law. We assume that the control instance satisfies

$$
\begin{aligned}
& t_{0}<\tau_{1}<\cdots \cdots<\tau_{k}<\tau_{k+1} \cdots, \\
& \lim _{k \longrightarrow \infty} \tau_{k}=\infty .
\end{aligned}
$$

A continuous nonlinear function $\phi(x): \mathbb{R}^{n} \longrightarrow \mathbb{R}^{n}$ stratifies $\phi(t, 0)=0$ and $\|\phi(x)\| \leq L\|x\|$, where $L$ is a Lipschitz constant. Many researchers have paid more attention on control system (1) and achieved many sufficient conditions for the stability of these systems [14-20]. Feng et al. consider single state-jumps impulsive systems with periodically time windows and give stability criteria for the new model [21]. To make the nonlinear impulse control system more reasonable, parameter uncertainty and bounded gain error are introduced into the corresponding impulsive differential equations [22-25]. Considering the limitation of machine and technology, Ma et al. investigate stabilization of impulse control systems with gain error and obtain a sufficient criterion for global exponential stability [26]. Zou et al. study impulsive systems with bounded gain error and form a sufficient criterion for the stability [27].

Cauchy-Schwarz inequality is an important tool to study nonlinear systems [28-31]. Recently, Peng et al. generalize the Cauchy-Schwarz inequality, which is used to deduce asymptotic stability for a class of nonlinear control systems [30]. Under the assumption $U(k, x)=B C x$, they study the after nonlinear system: 


$$
\left\{\begin{array}{l}
\dot{x}(t)=A x+\phi(x), t \neq \tau_{k}, \\
\Delta x=B C x, t=\tau_{k}, \quad k=1,2, \ldots, \\
x\left(t_{0}\right)=x_{0},
\end{array}\right.
$$

where $B$ and $C$ are constant matrixes. Based on the generalized Cauchy-Schwarz inequality, we consider a class of nonlinear impulsive control systems with the parameter uncertainty, which can be written as follows:

$$
\left\{\begin{array}{l}
\dot{x}(t)=(A+\Delta A) x+\phi(x), t \neq \tau_{k}, \\
\Delta x=B C x, t=\tau_{k}, \quad k=1,2, \ldots, \\
x\left(t_{0}\right)=x_{0} .
\end{array}\right.
$$

Generally, one can express the parameter uncertainty as $\Delta A=G F(t) H$ with $F^{T}(t) F(t) \leq I$. Here, matrixes $G$ and $H$ are given with appropriate dimensions. In this paper, we will find some conditions for the stability of system (4). We organize the paper as follows. In Section 2, we briefly introduce some related lemmas. Then, we show sufficient conditions in Section 3. The simulation experiment is shown in Section 4, and conclusion is listed in Section 4.

\section{Related Lemmas}

First of all, we introduce some lemmas to be used later. Throughout this paper, $\lambda_{\max }$ and $\lambda_{\min }$ are denoted as the largest eigenvalue and the smallest eigenvalue, respectively. $\|\cdot\|$ is denoted as the Euclidian norm of matric or vector.

Lemma 1 (see [30]). Suppose that $P$ is positive definite. If $x, y \in R^{n} \quad$ satisfy $\left|x^{T} y\right| \leq \sigma\left(x^{T} x\right)\left(y^{T} y\right)$ for a certain $\sigma \in[0,1]$, then

$$
\left(x^{T} P y\right)^{2} \leq\left(\frac{\lambda_{\max }(P)-g(\sqrt{\sigma}) \lambda_{\min }(P)}{\lambda_{\max }(P)+g(\sqrt{\sigma}) \lambda_{\min }(P)}\right)^{2}\left(x^{T} P x\right)\left(y^{T} P y\right)
$$

where $g(\sigma)=(1-\sigma / 1+\sigma)$.

Lemma 2 (see [27]). Suppose that $Q$ is symmetric and positive definite; then, for any $A, B \in R^{n \times n}$ and $\mu>0$,

$$
A^{T} \mathrm{Q} B+B^{T} \mathrm{Q} A \leq \mu A^{T} \mathrm{Q} A+\frac{1}{\mu} B^{T} \mathrm{Q} B .
$$

Lemma 3 (see [32]). Suppose that $H$ is a real symmetric matrix; then,

$$
\lambda_{\min }(H) x^{T} x \leq x^{T} H x \leq \lambda_{\max }(H) x^{T} x .
$$

\section{The Proposed Results}

We give the main results in this section. Specifically, we will analyze the stabilization of impulsive control system (4) with bounded gain error and parameter uncertainty and then list some sufficient conditions which assure the origin of the related systems is asymptotically stable.

Theorem 1. Suppose $P \in \mathbb{R}^{n \times n}$ be a symmetric and positive definite matrix, $\lambda_{1}=\lambda_{\min }(P), \lambda_{2}=\lambda_{\max }(P), I$ be the identity matrix, $\lambda_{3}$ be the largest eigenvalue of $P^{-1}\left(P A+A^{T} P\right)$, and $\lambda_{4}$ be the largest eigenvalue of the matrix $P^{-1}(I+B C)^{T} P(I+B C)$. If

$$
\left|x^{T}(t) \phi(x(t))\right| \leq \sigma\left(x^{T}(t) x(t)\right)\left(\phi(x(t))^{T} \phi(x(t))\right),
$$

for a certain $\sigma \in[0,1]$ and

$$
\left(\lambda_{3}+2 \sqrt{\left(\frac{\lambda_{2} \lambda_{\max }\left(G^{T} G\right) \lambda_{\max }\left(H^{T} H\right)}{\lambda_{1}}\right)}+2 L \frac{\lambda_{2}-g(\sqrt{\sigma}) \lambda_{1}}{\lambda_{2}+g(\sqrt{\sigma}) \lambda_{1}} \sqrt{\frac{\lambda_{2}}{\lambda_{1}}}\right)\left(\tau_{k+1}-\tau_{k}\right) \leq-\ln \left(\gamma \lambda_{4}\right),
$$

where

$$
g(\sigma)=\frac{1-\sigma}{1+\sigma}, \gamma>1
$$

then, we obtain that the origin of impulsive control system (4) is asymptotically stable.
Proof. We choose the Lyapunov function as follows:

$$
V(x(t))=x^{T}(t) P x(t) .
$$

When $t \neq \tau_{k}$, we obtain Dini's derivative of $V(x(t))$ for impulsive control system (4) as follows:

$$
\begin{aligned}
D^{+} V(x(t)) & =2 x^{T}(t) P((A+\Delta A) x(t)+\phi(x(t))), \\
& =2 x^{T}(t) P A x(t)+2 x^{T}(t) P \Delta A x(t)+2 x^{T}(t) P \phi(x(t)) .
\end{aligned}
$$


Next, we will calculate the three parts of the above formula (12), respectively. The matrices $P^{-1}\left(P A+A^{T} P\right)$ and $P^{-0.5}\left(P A+A^{T} P\right) P^{-0.5}$ have the same eigenvalues. By Lemma 3, we have

$$
\begin{aligned}
2 x^{T}(t) P A x(t) & =x^{T}(t)\left(P A+A^{T} P\right) x(t), \\
& =\left(x^{T}(t) P^{0.5}\right)\left(P^{-0.5}\left(P A+A^{T} P\right) P^{-0.5}\right)\left(P^{0.5} x(t)\right), \\
& \leq \lambda_{3}\left(x^{T}(t) P^{0.5}\right)\left(P^{0.5} x(t)\right), \\
& =\lambda_{3} V(x(t)) .
\end{aligned}
$$

According to the Cauchy-Schwarz inequality, we obtain $x^{T}(t) P \Delta A x(t) \leq \sqrt{\left(x^{T}(t) P^{2} x(t)\right)\left(x^{T}(t) \Delta A^{T} \Delta A x(t)\right)}$.

Since parameter uncertainty $\triangle A=G F(t) H$ and $F^{T}(t) F(t) \leq I$, inequality (14) can be rewritten as

$$
\begin{aligned}
2 x^{T}(t) P \Delta A x(t) & \leq 2 \sqrt{\left(x^{T}(t) P^{2} x(t)\right)\left(x^{T}(t) H^{T} F^{T}(t) G^{T} G F(t) H x(t)\right)}, \\
& \leq 2 \sqrt{\left(\left(x^{T}(t) P^{1 / 2}\right) P\left(P^{1 / 2} x(t)\right)\right)\left(x^{T}(t) H^{T} F^{T}(t) G^{T} G F(t) H x(t)\right)}, \\
& \leq 2 \sqrt{\left(\lambda_{2} V((x(t)))\right)\left(\lambda_{\max }\left(G^{T} G\right) x^{T}(t) H^{T} I H x(t)\right)}, \\
& \leq 2 \sqrt{\left(\lambda_{2} V(x(t))\right)\left(\lambda_{\max }\left(G^{T} G\right) \lambda_{\max }\left(H^{T} H\right) x^{T}(t) x(t)\right)}, \\
& =2 \sqrt{\left(\lambda_{2} V(x(t))\right)\left(\lambda_{\max }\left(G^{T} G\right) \lambda_{\max }\left(H^{T} H\right)\left(x^{T}(t) P^{1 / 2}\right) P^{-1}\left(P^{(1 / 2)} x(t)\right)\right)}, \\
& \leq 2 \sqrt{\left(\frac{\lambda_{2} \lambda_{\max }\left(G^{T} G\right) \lambda_{\max }\left(H^{T} H\right)}{\lambda_{1}}\right) V(x(t)) .}
\end{aligned}
$$

According to Lemma 1, we obtain

$$
\begin{aligned}
2 x^{T}(t) P \phi(x(t)) & \leq 2 L \frac{\lambda_{2}-g(\sqrt{\sigma}) \lambda_{1}}{\lambda_{2}+g(\sqrt{\sigma}) \lambda_{1}} \sqrt{\left(x^{T}(t) P x(t)\right)\left(\phi(x(t))^{T} P \phi(x(t))\right)}, \\
& \leq 2 L \frac{\lambda_{2}-g(\sqrt{\sigma}) \lambda_{1}}{\lambda_{2}+g(\sqrt{\sigma}) \lambda_{1}} \sqrt{\lambda_{2}\left(x^{T}(t) P x(t)\right)\left(\phi(x(t))^{T} \phi(x(t))\right)} .
\end{aligned}
$$

Since $\|\phi(x)\| \leq L\|x\|$, inequality (16) can be obtained as follows:

$$
\begin{aligned}
2 x^{T}(t) P \phi(x(t)) & \leq 2 L \frac{\lambda_{2}-g(\sqrt{\sigma}) \lambda_{1}}{\lambda_{2}+g(\sqrt{\sigma}) \lambda_{1}} \sqrt{\lambda_{2}\left(x^{T}(t) P x(t)\right)\left(x(t)^{T} x(t)\right)}, \\
& \leq 2 L \frac{\lambda_{2}-g(\sqrt{\sigma}) \lambda_{1}}{\lambda_{2}+g(\sqrt{\sigma}) \lambda_{1}} \sqrt{\lambda_{2}\left(x^{T}(t) P x(t)\right)\left(x(t)^{T} x(t)\right)}, \\
& \leq 2 L \frac{\lambda_{2}-g(\sqrt{\sigma}) \lambda_{1}}{\lambda_{2}+g(\sqrt{\sigma}) \lambda_{1}} \sqrt{\left(\frac{\lambda_{2}}{\lambda_{1}}\right)\left(x^{T}(t) P x(t)\right)\left(x(t)^{T} P x(t)\right),} \\
& =2 L \frac{\lambda_{2}-g(\sqrt{\sigma}) \lambda_{1}}{\lambda_{2}+g(\sqrt{\sigma}) \lambda_{1}} \sqrt{\frac{\lambda_{2}}{\lambda_{1}} V(x(t)) .}
\end{aligned}
$$


Combining inequalities (13), (15), and (17), we obtain

$$
D^{+} V(x(t)) \leq\left(\lambda_{3}+2 \sqrt{\left(\frac{\lambda_{2} \lambda_{\max }\left(G^{T} G\right) \lambda_{\max }\left(H^{T} H\right)}{\lambda_{1}}\right)}+2 L \frac{\lambda_{2}-g(\sqrt{\sigma}) \lambda_{1}}{\lambda_{2}+g(\sqrt{\sigma}) \lambda_{1}} \sqrt{\frac{\lambda_{2}}{\lambda_{1}}}\right) V(x(t)) .
$$

When $t=\tau_{k}$, we compute the value of $V$ as follows:

$$
\begin{aligned}
\left.V(x(t)+B C x(t))\right|_{t=\tau_{k}} & =\left.(x(t)+B C x(t))^{T} P(x(t)+B C x(t))\right|_{t=\tau_{k}}, \\
& =\left.x(t)^{T}(I+B C)^{T} P(I+B C) x(t)\right|_{t=\tau_{k}}, \\
& =\left.\left(x^{T}(t) P^{0.5}\right)\left(P^{-0.5}(I+B C)^{T} P(I+B C) P^{-0.5}\right)\left(P^{0.5} x(t)\right)\right|_{t=\tau_{k}} .
\end{aligned}
$$

It is known that the matrix with the matrix $P^{-1}(I+B C)^{T} P(I+B C)$. Thus, it follows $P^{-0.5}(I+B C)^{T} P(I+B C) P^{-0.5}$ has the same eigenvalues from (19) that

$$
\begin{aligned}
\left.V(x(t)+B C x(t))\right|_{t=\tau_{k}} & \leq\left.\lambda_{4}\left(x^{T}(t) P^{0.5}\right)\left(P^{0.5} x(t)\right)\right|_{t=\tau_{k}}, \\
& =\left.\lambda_{4} V(x(t))\right|_{t=\tau_{k}} .
\end{aligned}
$$

Now, we analyze the following comparison system:

$$
\begin{aligned}
\dot{\omega} & =\left(\lambda_{3}+2 \sqrt{\left(\frac{\lambda_{2} \lambda_{\max }\left(G^{T} G\right) \lambda_{\max }\left(H^{T} H\right)}{\lambda_{1}}\right)}+2 L \frac{\lambda_{2}-g(\sqrt{\sigma}) \lambda_{1}}{\lambda_{2}+g(\sqrt{\sigma}) \lambda_{1}} \sqrt{\frac{\lambda_{2}}{\lambda_{1}}}\right) \omega(t), t \neq \tau_{k}, \\
\omega\left(\tau_{k}^{+}\right) & =\lambda_{4} \omega\left(\tau_{k}\right), \\
\omega\left(\tau_{0}^{+}\right) & =\omega_{0} \geq 0 .
\end{aligned}
$$

According to the related conclusion (see Theorem 3 in [29]), we obtain that if

$$
\int_{\tau_{k}}^{\tau_{k+1}}\left(\lambda_{3}+2 \sqrt{\left(\frac{\lambda_{2} \lambda_{\max }\left(G^{T} G\right) \lambda_{\max }\left(H^{T} H\right)}{\lambda_{1}}\right)}+2 L \frac{\lambda_{2}-g(\sqrt{\sigma}) \lambda_{1}}{\lambda_{2}+g(\sqrt{\sigma}) \lambda_{1}} \sqrt{\frac{\lambda_{2}}{\lambda_{1}}}\right) \mathrm{d} t+\ln \left(\gamma \lambda_{4}\right) \leq 0, \gamma>1
$$

The origin of impulsive control system (4) is asymptotically stable.

Remark 1. If the parameter uncertainty $\Delta A=0$, the condition of (9) became the result of Theorem 3.1 in reference
[30]. Thus, the proposed method is a generalization of Peng's method.

In many practical applications, it is inevitable to put impulses with errors due to the limitation of machine and technology. So, we integrate the bounded gain error into the impulsive system (4). For simplicity, let $D=B C$. We rewrite 
the corresponding system as

$$
\left\{\begin{array}{l}
x(t)=(A+\Delta A) x(t)+\phi(x(t)), t \neq \tau_{k} \\
\Delta x(t)=(D+\Delta D) x(t), t=\tau_{k}, \quad k=1,2, \ldots \\
x\left(t_{0}\right)=x_{0}
\end{array}\right.
$$

where $\Delta D$ denotes the bounded gain error and has the following form: $\Delta D=m F(t) D$ with $m>0$ and $F^{T}(t) F(t) \leq I$. It is easy to obtain a similar analysis from Theorem 1 .
Theorem 2. Let $P \in \mathbb{R}^{n \times n}$ be a symmetric and positive definite matrix, $\lambda_{1}=\lambda_{\min }(P), \lambda_{2}=\lambda_{\max }(P), I$ be the identity matrix, and $\lambda_{3}$ be the largest eigenvalue of $P^{-1}\left(P A+A^{T} P\right)$. If

$$
\left|x^{T}(t) \phi(x(t))\right| \leq \sigma\left(x^{T}(t) x(t)\right)\left(\phi(x(t))^{T} \phi(x(t))\right) .
$$

for a certain $\sigma \in[0,1]$ and

$$
\left(\lambda_{3}+2 \sqrt{\left(\frac{\lambda_{2} \lambda_{\max }\left(G^{T} G\right) \lambda_{\max }\left(H^{T} H\right)}{\lambda_{1}}\right)}+2 L \frac{\lambda_{2}-g(\sqrt{\sigma}) \lambda_{1}}{\lambda_{2}+g(\sqrt{\sigma}) \lambda_{1}} \sqrt{\frac{\lambda_{2}}{\lambda_{1}}}\right)\left(\tau_{k+1}-\tau_{k}\right) \leq-\ln \left(\gamma \lambda_{4}\right),
$$

where

$$
\begin{gathered}
\lambda_{4}=\frac{\lambda_{2}}{\lambda_{1}}\left((1+\mu) \lambda_{\max }\left((I+D)^{T}(I+D)\right)+\left(1+\frac{1}{\mu}\right) m^{2} \lambda_{\max }\left(D^{T} D\right)\right), \\
g(\sigma)=\frac{1-\sigma}{1+\sigma}, \gamma>1 .
\end{gathered}
$$

$$
V(x(t))=x^{T}(t) P x(t) .
$$

Then, the origin of impulsive control system (23) is asymptotically stable.

Proof. We choose the following Lyapunov function as follows:
According to inequality (18), Dini's derivative of $V(x(t))$ for impulsive control system (23) is acquired as follows:

$$
D^{+} V(x(t)) \leq\left(\lambda_{3}+2 \sqrt{\left(\frac{\lambda_{2} \lambda_{\max }\left(G^{T} G\right) \lambda_{\max }\left(H^{T} H\right)}{\lambda_{1}}\right)}+2 L \frac{\lambda_{2}-g(\sqrt{\sigma}) \lambda_{1}}{\lambda_{2}+g(\sqrt{\sigma}) \lambda_{1}} \sqrt{\frac{\lambda_{2}}{\lambda_{1}}}\right) V(x(t)) .
$$

Then, we just need to compute We perform some calculations on $\left.V(x(t)+(D+\Delta D) x(t))\right|_{t=\tau_{k}}$. $\left.V(x(t)+(D+\Delta D) x(t))\right|_{t=\tau_{k}}$ and obtain

$$
\begin{aligned}
\left.V(x(t)+(D+\Delta D) x(t))\right|_{t=\tau_{k}} & =\left.(x(t)+(D+\Delta D) x(t))^{T} P(x(t)+(D+\Delta D) x(t))\right|_{t=\tau_{k}}, \\
& =\left.x(t)^{T}((I+D)+\Delta D)^{T} P((I+D)+\Delta D) x(t)\right|_{t=\tau_{k}}, \\
& \leq\left.\lambda_{2} x(t)^{T}((I+D)+\Delta D)^{T}((I+D)+\Delta D) x(t)\right|_{t=\tau_{k}} \\
& \leq\left.\lambda_{2} x(t)^{T}\left((I+D)^{T}(I+D)+(I+D)^{T} \Delta D+\Delta D^{T}(I+D)+\Delta D^{T} \Delta D\right) x(t)\right|_{t=\tau_{k}} .
\end{aligned}
$$


By using Lemma 2 and $\Delta D=m F(t) D$, we rewrite inequality (30) as

$$
\begin{aligned}
\left.V(x(t)+(D+\Delta D) x(t))\right|_{t=\tau_{k}} & \leq\left.\lambda_{2} x^{T}(t)\left((I+D)^{T}(I+D)+(I+D)^{T} \Delta D+\Delta D^{T} \Delta D+\Delta D^{T}(I+D)\right) x(t)\right|_{t=\tau_{k}} \\
& \leq\left.\lambda_{2} x^{T}(t)\left((1+\mu)(I+D)^{T}(I+D)+\left(1+\frac{1}{\mu}\right) \Delta D^{T} \Delta D\right) x(t)\right|_{t=\tau_{k}} \\
& =\left.\lambda_{2} x^{T}(t)\left((1+\mu)(I+D)^{T}(I+D)+\left(1+\frac{1}{\mu}\right) m^{2} D^{T} F^{T}(t) F(t) D\right) x(t)\right|_{t=\tau_{k}}
\end{aligned}
$$

It follows from (15) that

$$
x^{T}(t) x(t)=\left(x^{T}(t) P^{1 / 2}\right) P^{-1}\left(P^{1 / 2} x(t)\right) \leq \frac{V(x(t))}{\lambda_{1}} \text {. }
$$

Combine inequalities (31) and (32) and $F^{T}(t) F(t) \leq I$, we obtain

$$
\begin{aligned}
\left.V(x(t)+(D+\Delta D) x(t))\right|_{t=\tau_{k}} & =\left.\lambda_{2} x^{T}(t)\left((1+\mu)(I+D)^{T}(I+D)+\left(1+\frac{1}{\mu}\right) m^{2} D^{T} F^{T}(t) F(t) D\right) x(t)\right|_{t=\tau_{k}}, \\
& \leq\left.\lambda_{2} x^{T}(t)\left((1+\mu)(I+D)^{T}(I+D)+\left(1+\frac{1}{\mu}\right) m^{2} D^{T} D\right) x(t)\right|_{t=\tau_{k}}, \\
& \leq\left.\frac{\lambda_{2}}{\lambda_{1}}\left((1+\mu) \lambda_{\max }\left((I+D)^{T}(I+D)\right)+\left(1+\frac{1}{\mu}\right) m^{2} \lambda_{\max }\left(D^{T} D\right)\right) V(x(t))\right|_{t=\tau_{k}}, \\
& =\left.\lambda_{4} V(x(t))\right|_{t=\tau_{k} .}
\end{aligned}
$$

Here, we emit the rest analysis process, which is similar to Theorem 1. Thus, from equalities (29) and (33), we obtain that if

$$
\begin{aligned}
& \left(\lambda_{3}+2 \sqrt{\left(\frac{\lambda_{2} \lambda_{\max }\left(G^{T} G\right) \lambda_{\max }\left(H^{T} H\right)}{\lambda_{1}}\right)}+2 L \frac{\lambda_{2}-g(\sqrt{\sigma}) \lambda_{1}}{\lambda_{2}+g(\sqrt{\sigma}) \lambda_{1}} \sqrt{\frac{\lambda_{2}}{\lambda_{1}}}\right)\left(\tau_{k+1}-\tau_{k}\right) \leq-\ln \left(\gamma \lambda_{4}\right), \\
& \lambda_{4}=\frac{\lambda_{2}}{\lambda_{1}}\left((1+\mu) \lambda_{\max }\left((I+D)^{T}(I+D)\right)+\left(1+\frac{1}{\mu}\right) m^{2} \lambda_{\max }\left(D^{T} D\right)\right),
\end{aligned}
$$

the origin of impulsive control system (23) is asymptotically stable. This completes the proof.

\section{A Numerical Example}

In this section, we perform the proposed model on a numerical example to display its effectiveness. The example is produced by Qi and Chen [33]. Let $x=\left[x_{1}, x_{2}, x_{3}\right]^{T}$, $\phi(x)=\left[x_{2} x_{3},-x_{1} x_{3}, x_{1} x_{2}\right]^{T}$, and

$$
A=\left[\begin{array}{ccc}
-a & a & 0 \\
c & -1 & 0 \\
0 & 0 & -b
\end{array}\right] \text {. }
$$




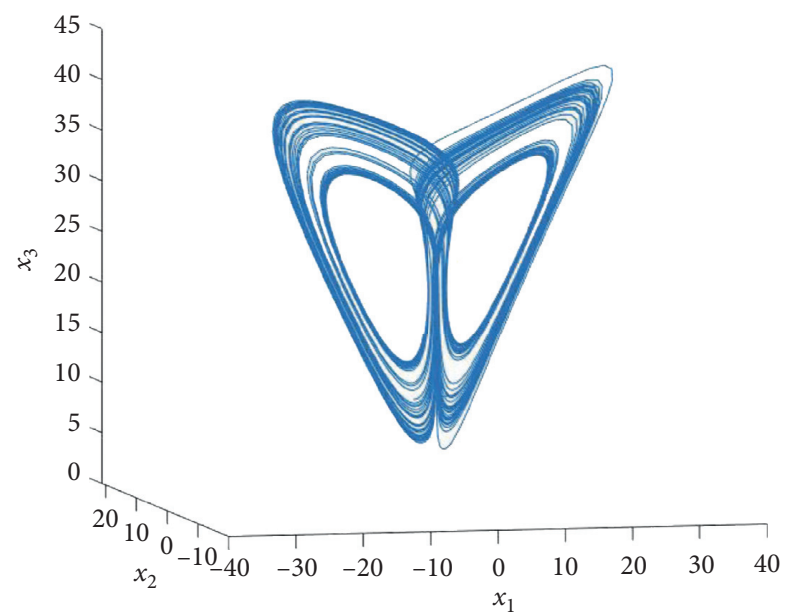

FIgURE 1: The chaotic phenomenon of system (36) with the initial condition: $x(0)=[3,5,10]^{T}$.

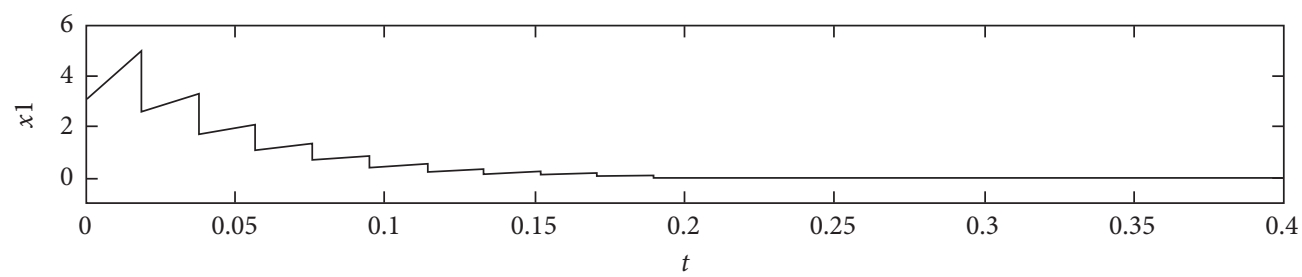

(a)

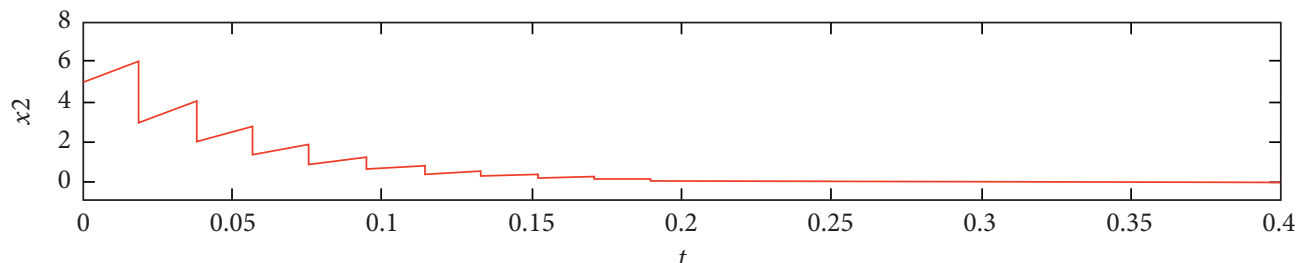

(b)

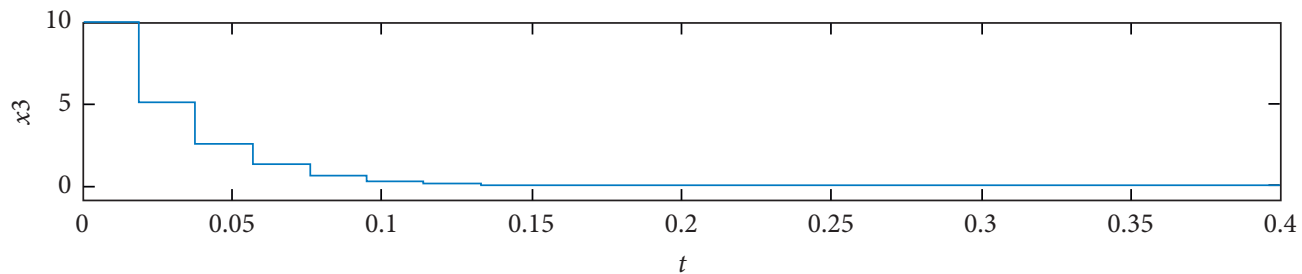

(c)

Figure 2: Time response curves for the controlled system (36) with the parameter uncertainty.

The corresponding state equation can be described as

$$
\dot{x}=A x+\phi(x) .
$$

According to the strategy of [33], some parameters of this system are set as $a=35, b=(8 / 3)$, and $c=25$. From Figure 1, we can see that system (36) is chaotic for the initial condition: $x(0)=[3,5,10]^{T}$.

After simple calculation, we obtain that

$$
\begin{aligned}
\|\phi(x)\| & =\sqrt{\left(x_{2} x_{3}\right)^{2}+\left(x_{1} x_{3}\right)^{2}+\left(x_{1} x_{2}\right)^{2},} \\
& \leq \max \left\{\left|x_{1}\right|,\left|x_{2}\right|,\left|x_{3}\right|\right\} \sqrt{x_{1}^{2}+x_{2}^{2}+x_{3}^{2}}, \\
& =\max \left\{\left|x_{1}\right|,\left|x_{2}\right|,\left|x_{3}\right|\right\}\|x\| .
\end{aligned}
$$

From Figure 1, we can intuitively find $\max \left\{\left|x_{1}\right|,\left|x_{2}\right|,\left|x_{3}\right|\right\} \leq 45$. Combining with inequality (37), the parameter $L$ can be set as 45 . Since 


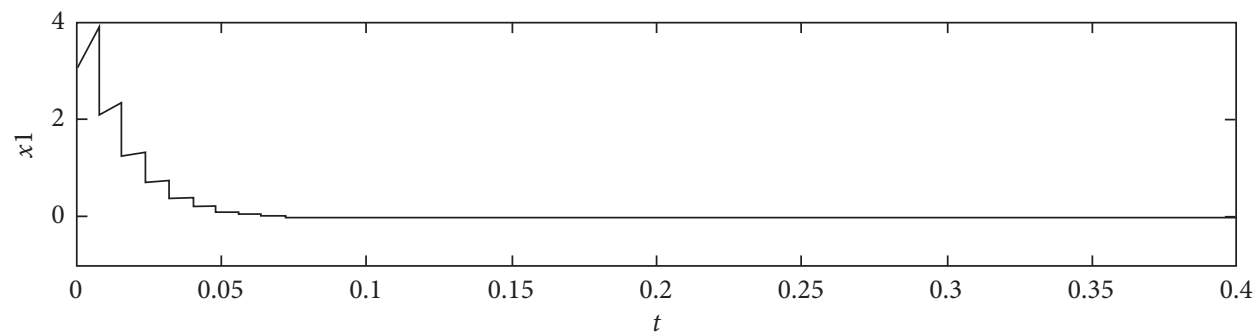

(a)

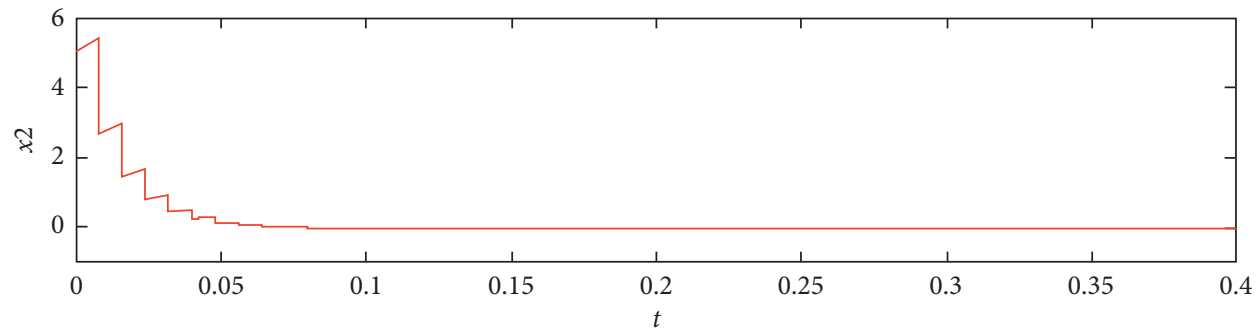

(b)

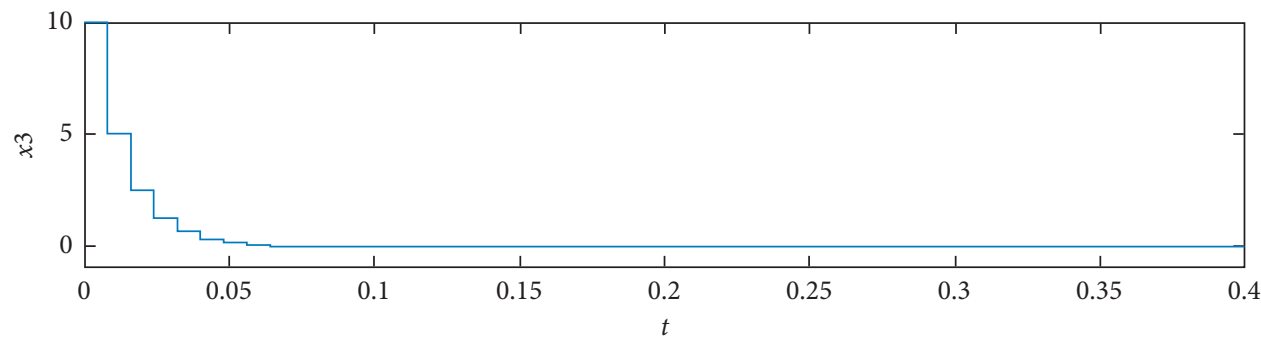

(c)

FIgURE 3: Time response curves for the controlled system (36) with parameter uncertainty and gain error.

$$
\left|x^{T} \phi(x)\right|^{2} \leq \frac{1}{9}\left(x^{T} x\right)\left(\phi(x)^{T} \phi(x)\right)
$$

the parameter $\sigma$ is chosen as $\sigma=(1 / 9)$. In this section, some matrices are chosen as follows:

$$
\begin{aligned}
& G=H=\left[\begin{array}{ccc}
0.5 & 0 & 0 \\
0 & 0.5 & 0 \\
0 & 0 & 0.5
\end{array}\right], \\
& P=C=\left[\begin{array}{lll}
1 & 0 & 0 \\
0 & 1 & 0 \\
0 & 0 & 1
\end{array}\right], \\
& B=\left[\begin{array}{ccc}
-0.5 & -0.01 & 0.02 \\
-0.01 & -0.5 & 0 \\
0.02 & 0 & -0.5
\end{array}\right] .
\end{aligned}
$$

Thus, the parameter uncertainty can be formed as

$$
\begin{aligned}
\Delta A= & {\left[\begin{array}{ccc}
0.5 & 0 & 0 \\
0 & 0.5 & 0 \\
0 & 0 & 0.5
\end{array}\right]\left[\begin{array}{ccc}
0.1 \sin (t) & 0 & 0 \\
0 & 0.1 \sin (t) & 0 \\
0 & 0 & 0.1 \sin (t)
\end{array}\right] } \\
& \cdot\left[\begin{array}{ccc}
0.5 & 0 & 0 \\
0 & 0.5 & 0 \\
0 & 0 & 0.5
\end{array}\right] .
\end{aligned}
$$

According to Theorem 1, we calculate $\lambda_{3}=32.9638$ and $\lambda_{4}=0.2729$. It follows from (8) that

$$
\tau_{k+1}-\tau_{k} \leq-\frac{\ln \left(\gamma \lambda_{4}\right)}{63.4638}
$$

If $\gamma=1.1$, it yields $\tau_{k+1}-\tau_{k} \leq 0.0190$. We choose $\tau_{k+1}-$ $\tau_{k}=0.0190$ and show the simulation result in Figure 2. The impulsive control system (36) is asymptotically stable. 
Next, we consider the controlled system (36) with the parameter uncertainty and the bounded gain error. The gain error is detailed as $\Delta D=m \sin (t) D$ in this section. We perform some similar calculation on (25) and obtain $\lambda_{3}=32.9638$. We choose $\mu=1$ and then obtain $\lambda_{4}=0.5458\left(1+m^{2}\right)$ from (26). Let $\gamma=1.1$ and $m=0.05$; then,

$$
\tau_{k+1}-\tau_{k} \leq 0.0080 .
$$

Thus, we choose $\tau_{k+1}-\tau_{k}=0.0080$ and show the experimental result in Figure 3. From this figure, we can obtain that the impulsive control system (36) is asymptotically stable.

\section{Conclusion}

We study the asymptotic stability of impulsive control systems with some uncertainty factors, such as the bounded gain error and the parameter. The proposed sufficient condition is established based on the generalized Cauchy-Schwarz inequality. We think the proposed issue is more practically applicable than some existing ones.

\section{Data Availability}

No data were used to support this study.

\section{Conflicts of Interest}

The authors declare that they have no conflicts of interest.

\section{Authors' Contributions}

All the authors contributed equally to write this paper. Z. Ren, S. Wen, and Y. Feng have proposed the main idea of the paper. Z. Ren, Q. Li, and N. Tang have proved the main theory. All authors read and approved the final manuscript.

\section{Acknowledgments}

This work was partially supported by the Chongqing Natural Science Foundation of China (cstc2019jcyj-msxmX0060), Key Project of Science and Technology Research Program of Chongqing Education Commission of China (KJZDK202001503), Key Project of Chongqing Municipal Key Laboratory of Institutions of Higher Education ([2017]3), National Natural Science Foundation of China (61601068 and 62061016), and Foundation of Chongqing Development and Reform Commission (2017[1007]).

\section{References}

[1] P. Naghshtabrizi, J. P. Hespanha, and A. R. Teel, "Exponential stability of impulsive systems with application to uncertain sampled-data systems," Systems \& Control Letters, vol. 57, no. 5, pp. $378-385,2008$.

[2] J. Sun, F. Qiao, and Q. Wu, "Impulsive control of a financial model," Physics Letters A, vol. 335, no. 4, pp. 282-288, 2005.

[3] X. L. Chai, Z. H. Gan, and C. X. Shi, "Impulsive synchronization and adaptive-impulsive synchronization of a novel financial hyperchaotic system," Mathematical Problems in Engineering, vol. 2013, Article ID 751616, , 2013.

[4] S. Gao, L. Chen, and Z. Teng, "Impulsive vaccination of an SEIRS model with time delay and varying total population size," Bulletin of Mathematical Biology, vol. 69, no. 2, pp. 731-745, 2007.

[5] Q. Liu and J. Wang, "A second-order multi-agent network for bound-constrained distributed optimization," IEEE Transactions on Automatic Control, vol. 60, no. 12, pp. 3310-3315, 2015.

[6] Z. G. Zeng and J. Wang, "Design and analysis of high-capacity associative memories based on a class of discrete-time recurrent neural networks," IEEE Transactions on Systems, Man, and Cybernetics, Part B (Cybernetics), vol. 38, no. 6, pp. 1525-1536, 2008.

[7] Y. Feng, X. Yang, Q. Song, and J. Cao, "Synchronization of memristive neural networks with mixed delays via quantized intermittent control," Applied Mathematics and Computation, vol. 339, pp. 874-887, 2018.

[8] X. Hu and L. Nie, "Exponential stability of nonlinear systems with impulsive effects and disturbance input," Advances in Difference Equations, vol. 2018, Article ID 354, 2018.

[9] Q. Song, H. Yan, Z. Zhao, and Y. Liu, "Global exponential stability of complex-valued neural networks with both timevarying delays and impulsive effects," Neural Networks, vol. 79, pp. 108-116, 2016.

[10] Y. Feng, X. Xiong, R. Tang, and X. Yang, "Exponential synchronization of inertial neural networks with mixed delays via quantized pinning control," Neurocomputing, vol. 310, no. 8, pp. 165-171, 2018.

[11] Q. Song, H. Yan, Z. Zhao, and Y. Liu, "Global exponential stability of impulsive complex-valued neural networks with both asynchronous time-varying and continuously distributed delays," Neural Networks, vol. 81, pp. 1-10, 2016.

[12] X. Yang, J. Cao, and Z. Yang, "Synchronization of coupled reaction-diffusion neural networks with time-varying delays via pinning-impulsive controller," SIAM Journal on Control and Optimization, vol. 51, no. 5, pp. 3486-3510, 2013.

[13] X. Yang, J. Lam, D. W. C. Ho, and Z. Feng, "Fixed-Time synchronization of complex networks with impulsive effects via nonchattering control," IEEE Transactions on Automatic Control, vol. 62, no. 11, pp. 5511-5521, 2017.

[14] J. Lu and D. W. C. Ho, "Globally exponential synchronization and synchronizability for general dynamical networks," IEEE Transactions on Systems, Man, and Cybernetics, Part B, vol. 40, no. 2, pp. 350-361, 2010.

[15] J. Lu, C. Ding, J. Lou, and J. Cao, “Outer synchronization of partially coupled dynamical networks via pinning impulsive controllers," Journal of the Franklin Institute, vol. 352, no. 11, pp. 5024-5041, 2015.

[16] X. Wang, C. Li, T. Huang, and X. Pan, "Impulsive control and synchronization of nonlinear system with impulse time window," Nonlinear Dynamics, vol. 78, no. 4, pp. 2837-2845, 2014.

[17] D. Yang, G. Qiu, and C. Li, "Global exponential stability of memristive neural networks with impulse time window and time-varying delays," Neurocomputing, vol. 171, pp. 10211026, 2016.

[18] J.-L. Wang, H.-N. Wu, and L. Guo, "Stability analysis of reaction-diffusion Cohen-Grossberg neural networks under impulsive control," Neurocomputing, vol. 106, pp. 21-30, 2013.

[19] J. Lu, D. W. C. Ho, and J. Cao, "A unified synchronization criterion for impulsive dynamical networks," Automatica, vol. 46, no. 7, pp. 1215-1221, 2010. 
[20] H. Wang, S. Duan, C. Li, L. Wang, and T. Huang, "Globally exponential stability of delayed impulsive functional differential systems with impulse time windows," Nonlinear Dynamics, vol. 84, no. 3, pp. 1655-1665, 2016.

[21] Y. Feng, C. Li, and T. Huang, "Periodically multiple statejumps impulsive control systems with impulse time windows," Neurocomputing, vol. 193, pp. 7-13, 2016.

[22] F. Chen, H. Wang, and C. Li, "Impulsive control of memristive chaotic systems with impulsive time window," Mathematical Problems in Engineering, vol. 2015, Article ID 927327, , 2015.

[23] H. Wang, S. Duan, C. Li, L. Wang, and T. Huang, "Stability criterion of linear delayed impulsive differential systems with impulse time windows," International Journal of Control, Automation and Systems, vol. 14, no. 1, pp. 174-180, 2016.

[24] X. Wang, J. Yu, C. Li, H. Wang, T. Huang, and J. Huang, "Robust stability of stochastic fuzzy delayed neural networks with impulsive time window," Neural Networks, vol. 67, pp. 84-91, 2015.

[25] Y. Zhou, C. Li, T. Huang, and X. Wang, "Impulsive stabilization and synchronization of Hopfield-type neural networks with impulse time window," Neural Computing and Applications, vol. 28, no. 4, pp. 775-782, 2017.

[26] T. Ma and F. Zhao, "Impulsive stabilization of a class of nonlinear system with bounded gain error," Chinese Physics B, vol. 23, no. 12, Article ID 150504, 2014.

[27] L. Zou, Y. Peng, Y. Peng, Y. Feng, and Z. Tu, "Impulsive control of nonlinear systems with impulse time window and bounded gain error," Nonlinear Analysis: Modelling and Control, vol. 23, no. 1, pp. 40-49, 2018.

[28] T. Yang, "Impulsive control," IEEE Transactions on Automatic Control, vol. 44, no. 5, pp. 1081-1083, 1999.

[29] T. Yang, :Impulsive Control Theory", Springer, Berlin, Germany, 2001.

[30] Y. Peng, J. Wu, L. Zou, Y. Feng, and Z. Tu, “A generalization of the cauchy-schwarz inequality and its application to stability analysis of nonlinear impulsive control systems," Complexity, vol. 2019, Article ID 6048909, 7 pages, 2019.

[31] X. L. Hu and J. Wang, "Solving pseudomonotone variational inequalities and pseudoconvex optimization problems using the projection neural network," IEEE Transactions on Neural Networks, vol. 17, no. 6, pp. 1487-1499, 2006.

[32] R. A. Horn and C. R. Johnson, "Matrix Analysis" Cambridge University Press, Cambrige, England, 1985.

[33] G. Qi, G. Chen, S. Du, Z. Chen, and Z. Yuan, "Analysis of a new chaotic system," Physica A: Statistical Mechanics and its Applications, vol. 352, no. 2-4, pp. 295-308, 2005. 